\title{
The role of water in the epoxidation over gold-titania catalysts
}

\author{
T. Alexander Nijhuis* and Bert M. Weckhuysen \\ Received (in Cambridge, UK) 8th September 2005, Accepted 11th October 2005 \\ First published as an Advance Article on the web 4th November 2005 \\ DOI: 10.1039/b512611h
}

Water is capable of reducing the rate of deactivation of goldtitania catalysts for propene epoxidation.

After our recent paper $^{1}$ examining the role of gold in gold-titania catalysts for propene epoxidation, we now focus on the role of water and water production. Gold-titania catalysts, at present, seem to be the only selective catalysts that can directly epoxidize propene. The production of water is a very important side reaction for these catalysts, and the hydrogen efficiency does, for a large part, determine the economics of the process. ${ }^{2,3}$ (The hydrogen efficiency is defined as the number of molecules of propene oxide produced divided by the number of hydrogen molecules consumed.) It has already been demonstrated that water has a significant rate-enhancing effect on $\mathrm{CO}$ oxidation over gold catalysts. ${ }^{4-6}$ However, for research into propene epoxidation, the influence of water has been largely ignored. In this paper the production of water over gold-titania catalysts is investigated, as well as the effect that water has on the epoxidation reaction.

Even though it is now 8 years since Haruta et al. ${ }^{7}$ discovered the exceptional capability of gold-titania catalysts for the epoxidation of propene, the mode of operation of these catalysts is still unknown. Gold-titania catalysts can epoxidize propene in the presence of a mixture of hydrogen and oxygen under very mild conditions (323-373 K and atmospheric pressure). The fact that both hydrogen and oxygen are needed for this oxidation reaction has triggered speculation that a peroxide species would be an important reaction intermediate for the oxidation, ${ }^{2,8,9}$ which is supported by the fact that titania is capable of epoxidizing propene using hydrogen peroxide in the liquid phase, ${ }^{10}$ and the fact that gold has been observed to produce hydrogen peroxide directly from hydrogen and oxygen. ${ }^{11}$ Although these gold-titania epoxidation catalysts are highly selective, they have two main problems that need to be addressed. The conversion remains quite low (typically below $2 \%$, although conversions of up to $10 \%$ have been reported with certain promoters ${ }^{12}$ ) and the hydrogen efficiency is insufficient, ${ }^{3}$ typically $30 \%$. The low product yield of gold-titania catalysts is usually explained by a strong adsorption of propene oxide onto the catalyst. ${ }^{1,3,12}$ The final aim of our research is to develop a reaction mechanism for this system that will help us to design a more active catalyst with a high hydrogen efficiency.

Highly dispersed gold on titania catalysts were prepared by a deposition-precipitation method. A solution of $\mathrm{HAuCl}_{4}$ in water was slowly added to a stirred suspension of P25 titania in water, the $\mathrm{pH}$ of which was kept at 9.5 using aqueous ammonia. After aging the catalyst for $30 \mathrm{~min}$, it was filtered and washed 3 times

Department for Inorganic Chemistry and Catalysis, Utrecht University, Sorbonnelaan 16, 3584CA, Utrecht, The Netherlands.

E-mail: x.nijhuis@chem.uu.nl; Fax: (+31) 30-2511027;

Tel: (+31) 30-2537763 with de-ionized water. The catalyst was dried in air overnight at $393 \mathrm{~K}$ and calcined for $4 \mathrm{~h}$ at $673 \mathrm{~K}\left(10 \mathrm{~K} \mathrm{~min}^{-1}\right.$ heating and cooling rate). TEM analysis showed a narrow major gold particle size distribution of 3.2-4.2 nm with few smaller or larger particles, the whole range being 2-6 nm. XRF analysis showed the amount of chlorine on the catalyst (a known promoter) to be lower than the detection limit of $7 \mathrm{ppm}$. The only contaminant present in a significant quantity was nickel (200 ppm), which was also present in the P25 used. Catalytic tests were performed in a flow setup. $0.3 \mathrm{~g}$ of catalyst was tested, typically with a gas feed rate of $50 \mathrm{ml} \mathrm{min}{ }^{-1}$ (STP) (Gas Hourly Space Velocity (GHSV) $9000 \mathrm{~h}^{-1}$ ), consisting of $10 \mathrm{vol} \%$ (each) of hydrogen, oxygen and propene in helium. Water vapor was optionally added by switching the gas feed through water in a saturator. The gas composition was analyzed using a fast Interscience Compact GC system (3 min analysis time) containing a Porabond Q column and a Molsieve $5 \mathrm{~A}$ column in two separate channels, each with a thermal conductivity detector.

In Fig. 1 and Fig. 2 the effect of water on the epoxidation, and the effect of the epoxidation on the water formation are shown, respectively. In the presence of a small quantity of water ( $1 \mathrm{vol} \%)$ the rate of the epoxidation remains almost unaffected, the main difference is the fact that propene oxide can be observed almost immediately as a reaction product. In the absence of water, there is a delay of about 30 min before propene oxide is detected as a product. This is attributed to a strong adsorption of propene oxide on the titania. ${ }^{13}$ All propene oxide produced remains adsorbed on the catalyst surface until it is almost completely covered with bidentate propoxy species, ${ }^{1,14}$ the species formed on titania upon propene oxide adsorption. In the presence of excess water ( $1 \mathrm{vol} \%$

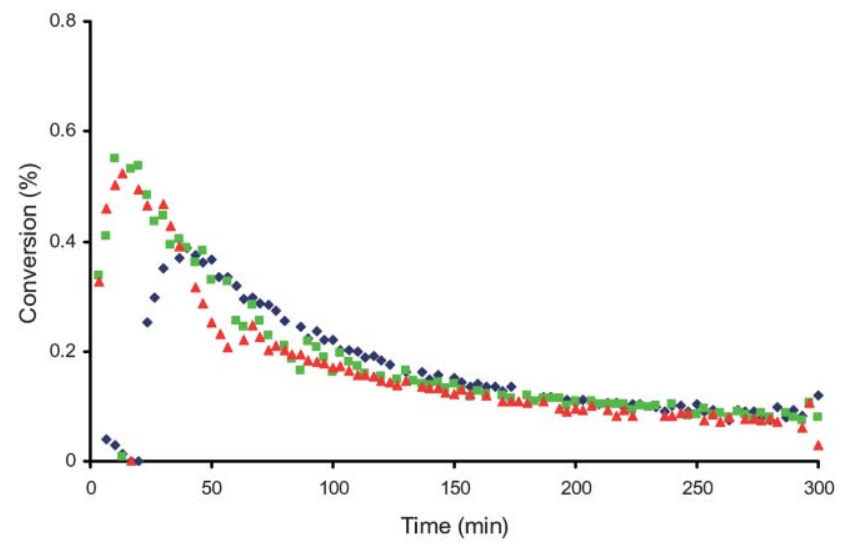

Fig. 1 Propylene conversion to propylene oxide in a dry gas stream $(\diamond)$, gas stream containing $1 \mathrm{vol} \%$ of water $(\mathbf{\Lambda})$, and a dry gas stream over a wetted catalyst (ם) $\left(1 \mathrm{wt} \% \mathrm{Au}\right.$ on $\mathrm{TiO}_{2}$ catalyst, gas feed $10 \%$ propene, $10 \%$ oxygen, $10 \%$ hydrogen in helium, $323 \mathrm{~K}, 1.1 \mathrm{bar}$, GHSV $9000 \mathrm{~h}^{-1}$ ). 


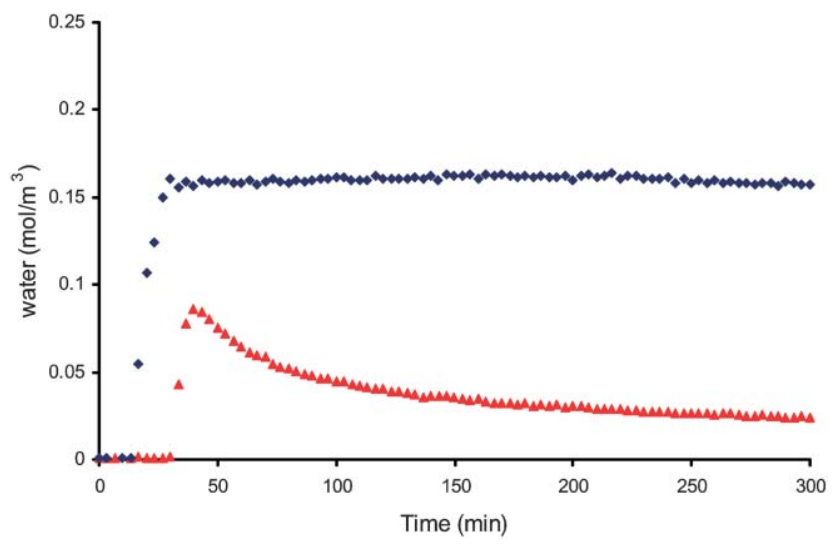

Fig. 2 Water production over a $1 \mathrm{wt} \% \mathrm{Au}$ on $\mathrm{TiO}_{2}$ catalyst during epoxidation $(\boldsymbol{\Lambda}, 10 \%$ propene, $10 \%$ oxygen, $10 \%$ hydrogen in helium) and hydrogen oxidation $(\bullet, 6 \%$ hydrogen and $6 \%$ oxygen in helium) (323 K, 1.1 bar, GHSV $9000 \mathrm{~h}^{-1}$ ).

is a large amount compared to the $0.05 \mathrm{vol} \%$ of propene oxide observed as the highest concentration in the gas phase), the propene oxide adsorbs relatively less due to the competitive adsorption of water. An almost identical propene oxide production curve to the one shown in Fig. 1 for the experiment in the presence of water is obtained from an experiment in which the catalyst is 'pre-wetted' prior to the oxidation reaction by performing a $30 \mathrm{~min}$ hydrogen oxidation (no propene present) with a $6 \%$ hydrogen and $6 \%$ oxygen mixture.

In Fig. 2 it can be seen that propene epoxidation has a large influence on the water formation rate. In the absence of propene, the water production rate is significantly higher (on average 4 times higher), even though lower hydrogen and oxygen concentrations were used (to circumvent working with explosive gas mixtures). When water production is the only reaction occurring, there is no catalyst deactivation, while during the epoxidation, the catalyst deactivates at the same rate for both the epoxidation and the water formation. This indicates that both reactions either share the same reaction site, or have a common reaction intermediate. The differences in rate can be explained by propene or propene oxide adsorbing onto a site used for the water production. The initial delay in water production for both experiments is explained by the strong adsorption of the produced water on the titania catalyst support.

The catalyst deactivation we observed during the epoxidation was easily reversed by a low temperature ( $573 \mathrm{~K}$ ) calcination, after which the catalyst regained its original activity. TEM and UV-vis analysis of the catalyst after use showed that it had not changed. The catalyst deactivation for this system has been attributed to different possible consecutive reactions of the propene oxide produced. Possible explanations reported in the literature for the deactivation are: oligomerization of propene oxide, ${ }^{2,9}$ irreversible propene oxide adsorption as a bidentate propoxy species, ${ }^{14}$ or oxidation of adsorbed propene oxide to carboxylates. ${ }^{1,13}$ No indications are available that changes to the gold particles, or adsorbed species on the gold particles, cause deactivation. For the propene epoxidation, both gold and titania are essential for the catalytic activity. In a study by Barton and Podkolzin ${ }^{15}$ on water production over gold catalysts, it was shown that all this reaction's important steps occur on the gold. However, it cannot be ruled out that one of the reaction/adsorption steps of water production includes a decomposition or adsorption occurring via the support, which could link the activity for water production to a deactivation occurring by the loss of sites on the titania support.

In Fig. 3, the results from an experiment, in which the epoxidation is carried out at a water concentration of $2 \mathrm{vol} \%$, are shown. In this experiment it can be seen that at a higher water concentration, both the propene epoxidation rate and the deactivation rate are lower. The first observation can be explained by lower propene adsorption on the titania as a consequence of the competitive adsorption of water. The lower deactivation rate can be attributed to lower propene oxide production and the linkage of the catalyst deactivation to propene oxide consecutive reactions on the titania, as discussed earlier.

Fig. 4 shows experiments in which the water addition to the gas feed was switched on or off halfway through a 5 hour catalytic cycle. It can be seen that the removal of water from the gas phase caused an increase in catalytic activity, while the addition of water caused a decrease. These observations can also be explained by the competitive adsorption of water and propene oxide, and the

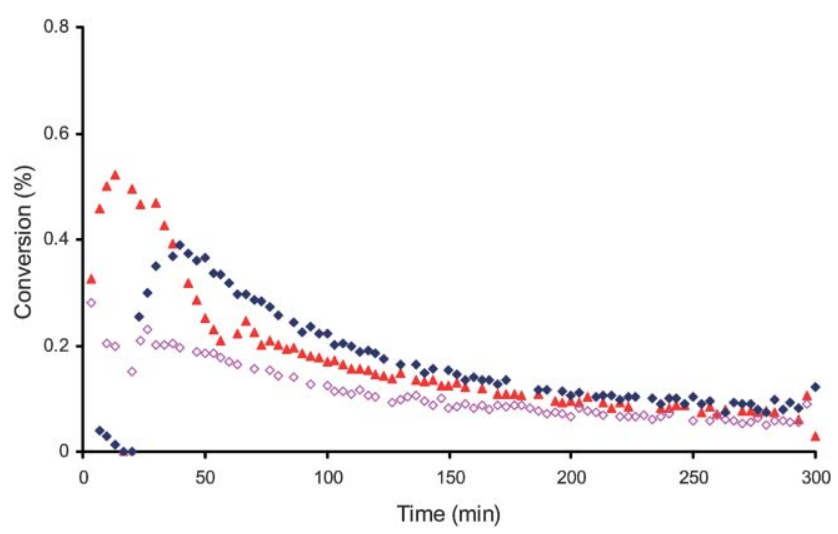

Fig. 3 Propylene conversion to propylene oxide in a gas stream containing different water concentrations: $0 \%(\bullet), 1 \mathrm{vol} \%(\mathbf{\Delta})$, and $2 \mathrm{vol} \%(\diamond)$. (1 wt $\%$ Au on $\mathrm{TiO}_{2}$ catalyst, gas feed $10 \%$ propene, $10 \%$ oxygen, $10 \%$ hydrogen in helium, $323 \mathrm{~K}, 1.1 \mathrm{bar}$, GHSV $9000 \mathrm{~h}^{-1}$ ).

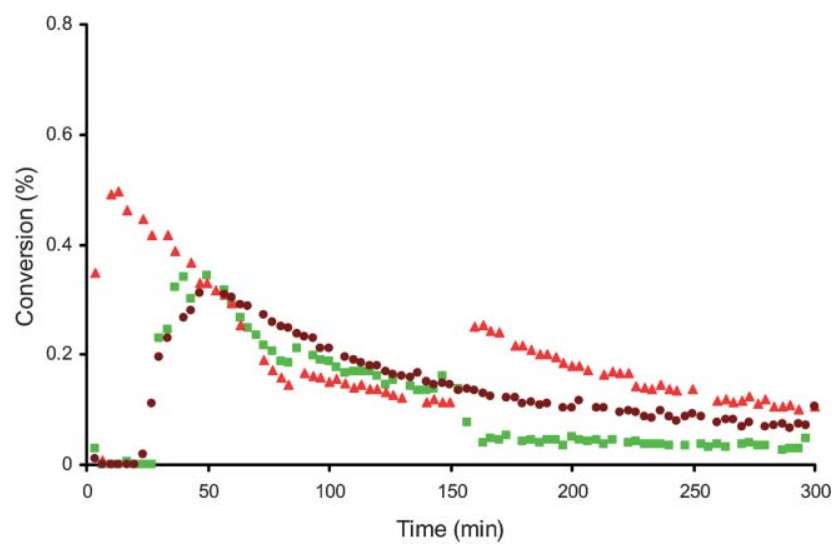

Fig. 4 Water addition during propene conversion to propene oxide over a $1 \mathrm{wt} \% \mathrm{Au}$ on $\mathrm{TiO}_{2}$ catalyst (gas feed $10 \%$ hydrogen, oxygen and propene in helium, 1.1 bar, $323 \mathrm{~K}$, GHSV $9000 \mathrm{~h}^{-1}$ ) $1 \mathrm{vol} \%$ of water added to gas feed: $\bullet$ no water added, $\boldsymbol{\Delta}$ water added from 0-150 min, $\boldsymbol{\square}$ water added from $150-300 \mathrm{~min}$. 
catalyst deactivation by propene oxide adsorbed on the titania. In the presence of water, the propylene oxide concentration on the catalyst is slightly lower, with both a slower reaction rate and a lower deactivation rate as a result. At a water level of $1 \mathrm{vol} \%$ these two effects balance with each other, causing a reaction rate similar to that when water is absent. At higher water concentrations these two separate effects are more clearly apparent. In experiments in which the water was added/removed halfway through a catalytic cycle (Fig. 4), they can also be distinguished separately. If the water is removed halfway through the experiment, the activity goes up, as there is no longer the competitive adsorption of water. The reaction rate in this case is higher than in the experiment in which water was not added, since the deactivation was less, due to a lower propylene oxide concentration on the catalyst. In an experiment in which water is added halfway through, the activity goes down significantly, since from that moment on, the reaction is hindered by competitive water adsorption. Zwijnenburg et al. ${ }^{3}$ recently reported that it is possible to desorb propene oxide from a gold-titania catalyst by starting a co-feed of water, methanol, or acetone. They similarly attributed this to competitive adsorption. However, in their experiments, no changes in deactivation or propylene oxide production rates were observed. This is probably caused by the addition of lower quantities of water in their work.

In an experiment, in which, during hydrogen oxidation, water was added to the gas feed stream and subsequently removed, it was observed that for the water production, the addition of water had no effect. This was also observed by Barton and Podkolzin. ${ }^{15}$ This indicates that, as expected, water adsorption on the gold particles is negligible, and that the effect of water on the epoxidation and deactivation rates is only via competitive adsorption effects on the titania.

It can be concluded that over gold-titania catalysts, propene epoxidation and water production have a strong influence on each other. Water addition to the feed gas stream is therefore recommended as it suppresses the catalyst deactivation by lowering the propene oxide concentration adsorbed on the titania through competitive adsorption. However, as a side effect, the presence of water causes a small decrease in the catalytic activity.

NWO/STW and NWO/CW are kindly acknowledged for their financial support (VIDI and VENI grants) of T. A. N. and B. M. W.

\section{Notes and references}

1 T. A. Nijhuis, T. Visser and B. M. Weckhuysen, Angew. Chem., Int. Ed., 2005, 44, 1115-1118.

2 T. A. Nijhuis, B. J. Huizinga, M. Makkee and J. A. Moulijn, Ind. Eng. Chem. Res., 1999, 38, 884-891.

3 A. Zwijnenburg, M. Makkee and J. A. Moulijn, Appl. Catal., A, 2004, 270, 49-56.

4 J. T. Calla and R. J. Davis, J. Phys. Chem. B, 2005, 109, 2307-2314.

5 C. K. Costello, J. Guzman, J. H. Yang, Y. M. Wang, M. C. Kung, B. C. Gates and H. H. Kung, J. Phys. Chem. B, 2004, 108, 12529-12536.

6 M. Date, M. Okumura, S. Tsubota and M. Haruta, Angew. Chem., Int. Ed., 2004, 43, 2129-2132.

7 T. Hayashi, K. Tanaka and M. Haruta, J. Catal., 1998, 178, 566-575.

8 C. Sivadinarayana, T. V. Choudhary, L. L. Daemen, J. Eckert and D. W. Goodman, J. Am. Chem. Soc., 2004, 126, 38-39.

9 E. E. Stangland, K. B. Stavens, R. P. Andres and W. N. Delgass, J. Catal., 2000, 191, 332-347.

10 M. G. Clerici, G. Bellussi and U. Romano, J. Catal., 1991, 129, 159-167.

11 P. Landon, P. J. Collier, A. J. Papworth, C. J. Kiely and G. J. Hutchings, Chem. Commun., 2002, 2058-2059.

12 A. K. Sinha, S. Seelan, S. Tsubota and M. Haruta, Angew. Chem., Int. Ed., 2004, 43, 1546-1548.

13 T. A. Nijhuis, T. Visser and B. M. Weckhuysen, J. Phys. Chem. B, 2005, 109, 19309-19319.

14 G. Mul, A. Zwijnenburg, B. van der Linden, M. Makkee and J. A. Moulijn, J. Catal., 2001, 201, 128-137.

15 D. G. Barton and S. G. Podkolzin, J. Phys. Chem. B, 2005, 109, 2262-2274. 